Vernehmlassung zur Verordnung über die Versichertenkarte:

\title{
Unausgereiftes Projekt des Bundesrates
}

\author{
Die FMH befürwortet grundsätzlich die Einführung einer Versichertenkarte. \\ Das Projekt, das der Bundesrat in seiner Vernehmlassung präsentiert, ist aller- \\ dings überhaupt nicht ausgereift - es muss unbedingt überprüft und ange- \\ passt werden.
}

Diese Stellungnahme hat die FMH am 26. Oktober 2006 als Medienmitteilung publiziert.
Es ist keineswegs gewährleistet, dass die vom Bundesrat deklarierten Ziele - effiziente Administration, Qualität und Sicherheit, Stärkung der Patientinnen und Patienten - mit der Karte, wie sie als Projekt vorliegt, erreicht werden.

Gerade der Schutz der Patientinnen und Patienten und ihrer Rechte muss gewährleistet sein - insbesondere, was die persönlichen elektronischen Daten anbelangt. Zum Beispiel unterliegt alleine die Tatsache, dass ein Patient einen Arzt - gleich welcher Fachrichtung - konsultiert, der ärztlichen Schweigepflicht und darf als Information den Versicherern nicht zugänglich gemacht werden.

Auch ist es nicht sinnvoll, dass eine Versichertenkarte medizinische Daten enthält: Angesichts der Freiwilligkeit der Einträge wüsste niemand, ob die Angaben stimmen und vollständig sind.
Damit würde für die Patientinnen und Patienten ein falsches und gefährliches Sicherheitsgefühl geschaffen.

Administrative Abläufe sollen vereinfacht werden - keinesfalls sollen hier zusätzliche Aufwände und Kosten geschaffen werden. So, wie es jetzt aussieht, entstehen für Ärztinnen und Ärzte jedoch erhebliche Mehrbelastungen: Pro Jahr und Praxis können die Mehrkosten laut einer Schätzung rund 25000 Franken betragen. Dazu kommen Probleme mit der Praktikabilität, die unannehmbar sind, weil sie mit dem normalen Betrieb einer Arztpraxis schlicht inkompatibel sind.

Fazit: Die vorliegende Verordnung ist überhaupt nicht ausgereift. Ein Marschhalt, der die nötigen Überprüfungen und Korrekturen erlaubt, ist deshalb dringend angesagt. 